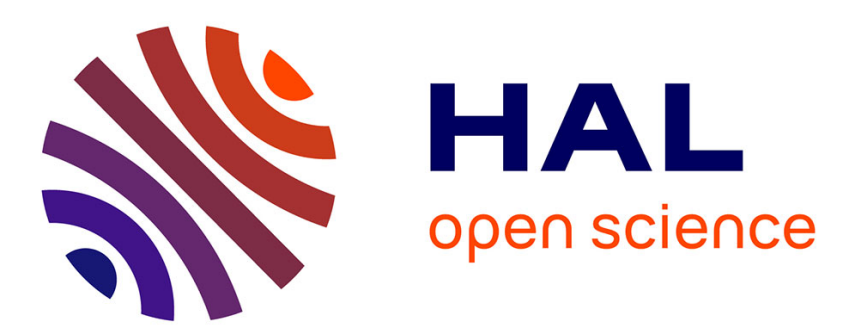

\title{
Microscopie X par réflexion totale et diffraction de Kossel à incidence rasante: premiers résultats
}

\author{
D. Erre, H. Jibaoui, J. Cazaux
}

\section{To cite this version:}

D. Erre, H. Jibaoui, J. Cazaux. Microscopie X par réflexion totale et diffraction de Kossel à incidence rasante: premiers résultats. Journal de Physique IV Proceedings, 1996, 06 (C4), pp.C4-393-C4-398. 10.1051/jp4:1996435 . jpa-00254318

\section{HAL Id: jpa-00254318 https://hal.science/jpa-00254318}

Submitted on 1 Jan 1996

HAL is a multi-disciplinary open access archive for the deposit and dissemination of scientific research documents, whether they are published or not. The documents may come from teaching and research institutions in France or abroad, or from public or private research centers.
L'archive ouverte pluridisciplinaire HAL, est destinée au dépôt et à la diffusion de documents scientifiques de niveau recherche, publiés ou non, émanant des établissements d'enseignement et de recherche français ou étrangers, des laboratoires publics ou privés. 


\title{
Microscopie X par réflexion totale et diffraction de Kossel à incidence rasante : premiers résultats
}

D. Erre, H. Jibaoui et J. Cazaux

LASSI, UFR Sciences, BP. 1039, 51687 Reims cedex 2, France

\begin{abstract}
Résumé : Un microscope électronique à balayage a été converti en microscope $\mathrm{X}$ par l'adjonction, entre autres, d'une caméra $\mathrm{CCD}$ qui permet d'acquérir rapidement des microradiographies $\mathrm{X}$ par projection ainsi que des diagrammes de Kossel par transmission. En modifiant ensuite les positions relatives de la source $\mathrm{X}$ et de l'objet par rapport à la caméra $\mathrm{CCD}$, nous montrons qu'il est possible d'obtenir successivement des diagrammes de diffraction par réflexion et des images topographiques agrandies de la surface par réflexion totale.
\end{abstract}

\begin{abstract}
A scanning electron microscope has been modified by the addition of a CCD camera in order to acquire rapidly either projection X-ray microradiographies or transmission Kossel diffraction patterns. By changing the relative position of the $X$-ray source and of the specimen with respect to the $C C D$ camera, we show that it is also possible to obtain successively reflection diffraction patterns and also magnified topographic images of the surface by X-ray total reflection.
\end{abstract}

\section{INTRODUCTION}

Il est bien connu que l'observation d'un échantillon par diffraction des rayons $\mathbf{X}$ à incidence presque rasante puis par réflexion totale apporte des informations inédites sur la structure cristalline et la topographie des premières couches atomiques des surfaces et interfaces ainsi analysées. La plupart de ces études sont conduites à l'aide d'appareils de diffraction de rayons $\mathrm{X}$ spécifiques à ce type d'étude [1-7]. Notre objectif est de montrer que des diagrammes à deux dimensions et des images de réflexion totale peuvent être rapidement obtenus en faisceau divergent dans un microscope électronique à balayage équipé d'une caméra $\mathrm{CCD}$. Ces travaux sinscrivent dans la logique de travaux antérieurs concernant la microscopie $\mathrm{X}$ analytique, la microtomographie et l'étude des phénomènes dynamiques qui ont aussi été conduits au laboratoire, mais par transmission, avec le même instrument basé sur des modifications légères apportées à un microscope électronique à balayage (MEB) [8-10].

Il s'agit ici d'indiquer les premiers résultats acquis très récemment dans un mode "par réflexion".

\section{L'INSTRUMENT ET SES MODIFICATIONS}

On trouvera par ailleurs le schéma de principe de l'instrument tel qu'il est mis en oeuvre en microscopie $X$ par projection et en microtomographie $X$ [8-10] et ses applications dans le domaine de la reconstruction 3D d'objets biologiques [11] aussi bien que de la cartographie 
élémentaire d'espèces en solution [12] qui font l'objet de contributions spécifiques à ce même congrès.

Dans la géométrie initiale, le faisceau d'électrons incidents bombarde une cible métallique mince $(\mathrm{Al}, \mathrm{Cu}$, etc...) pour générer un faisceau de rayons $\mathrm{X}$ divergent qui éclaire un écran convertisseur photon $X$ - photon visible suivie d'une caméra $C C D$ refroidie par effet Peltier. Tout échantillon interposé entre la source $\mathrm{X}$ quasiment ponctuelle et le convertisseur conduira à une image projetée sur ce convertisseur qui sera agrandie géométriquement en fonction du rapport des distances entre la source $X$ et le convertisseur d'une part et la source $X$ et l'échantillon d'autre part.

Cette image géométrique subit un deuxième grandissement électronique via le système d'acquisition et de restitution de cette image sur un écran vidéo ; l'intérêt de l'emploi de la caméra $C C D$ réside dans sa sensibilité, sa linéarité et sa grande dynamique ce qui conduit à des temps d'acquisition de quelques dizaines de secondes pour l'obtention de microradiographies $\mathrm{X}$ (d'absorption) de bonne qualité [8-12].

Si l'objet est monocristallin et d'épaisseur constante, l'absorption est uniforme et l'image acquise ne sera autre que le diagramme de pseudo-Kossel du cristal observé [13]. La figure 1 montre à titre d'exemple le diagramme obtenu à l'aide de notre dispositif avec un monocristal de fluorure de lithium de 0,46 millimètres d'épaisseur. Par rapport à l'emploi conventionnel de films photographiques on notera la relative rapidité d'acquisition d'un tel diagramme numérisé assortie d'une perte de résolution angulaire due à la dimension significative ( 22 microns) de chaque pixel de la caméra $\mathrm{CCD}$.
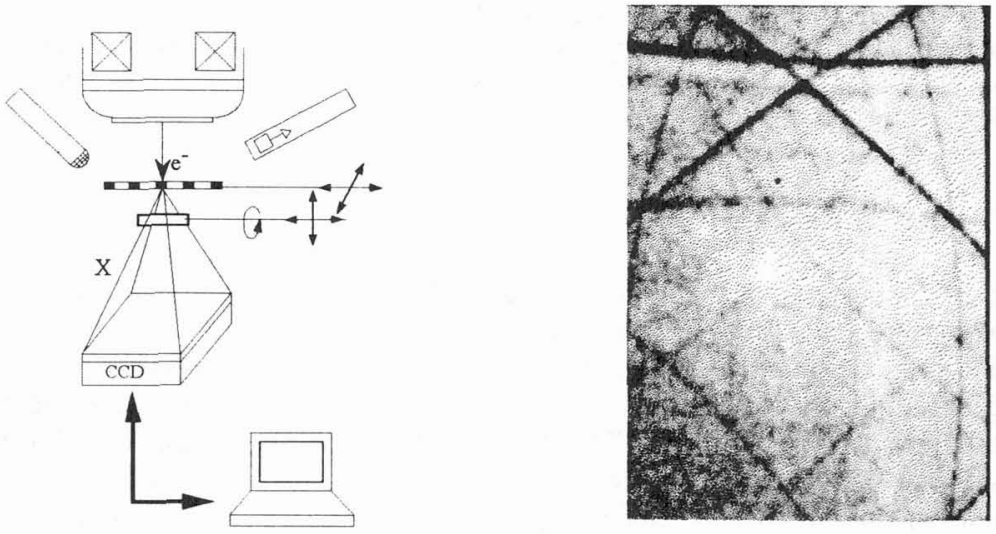

Figure 1 : A gauche : schéma de principe du dispositif qui, dans un MEB, permet l'obtention de microradiographies X par projection mais aussi de diagrammes de pseudo-Kossel de monocristaux. A droite : diagramme de pseudo-Kossel du LiF obtenu en 5 secondes à l'aide d'une anticathode de $\mathrm{Cu}$.

On the left : schematic drawing of the experimental arrangement implemented in a SEM which allows the acquisition of projection X-ray microradiographies but also, for single crystals, pseudo-Kossel patterns. On the right : LiF pseudo-Kossel pattern acquired in 5 seconds with the use of a $\mathrm{Cu}$ target.

Pour conduire les expériences par réflexion (imagerie et diffraction des rayons $\mathrm{X}$ ) nous avons d'abord songé à tourner l'échantillon de $90^{\circ}$ sans modifier ni la position de la source ni celle de la caméra. Cette géométrie a été rapidement abandonnée car elle conduit dans le plan de l'image à des grandissements différents suivant les directions parallèle ou perpendiculaire au plan de l'échantillon. En fait l'anamorphose significative de ces images les rendaient inexploitables. 
La version finalement retenue est représentée sur la figure 2. Elle consiste à rapprocher l'anticathode, source $X$, du plan de la caméra en collectant une partie des rayons $X$ générés par réflexion, l'échantillon étant placé légèrement au-dessus de cette source $X$ et sensiblement parallèle au plan de la caméra. On peut constater que ce dispositif conduit à un grandissement géométrique identique (de l'ordre de $10 \mathrm{ici}$, mais qui pourrait être accru en éloignant la caméra de l'échantillon) dans les 2 directions orthogonales du plan image si le plan de la caméra et l'échantillon sont strictement parallèles.

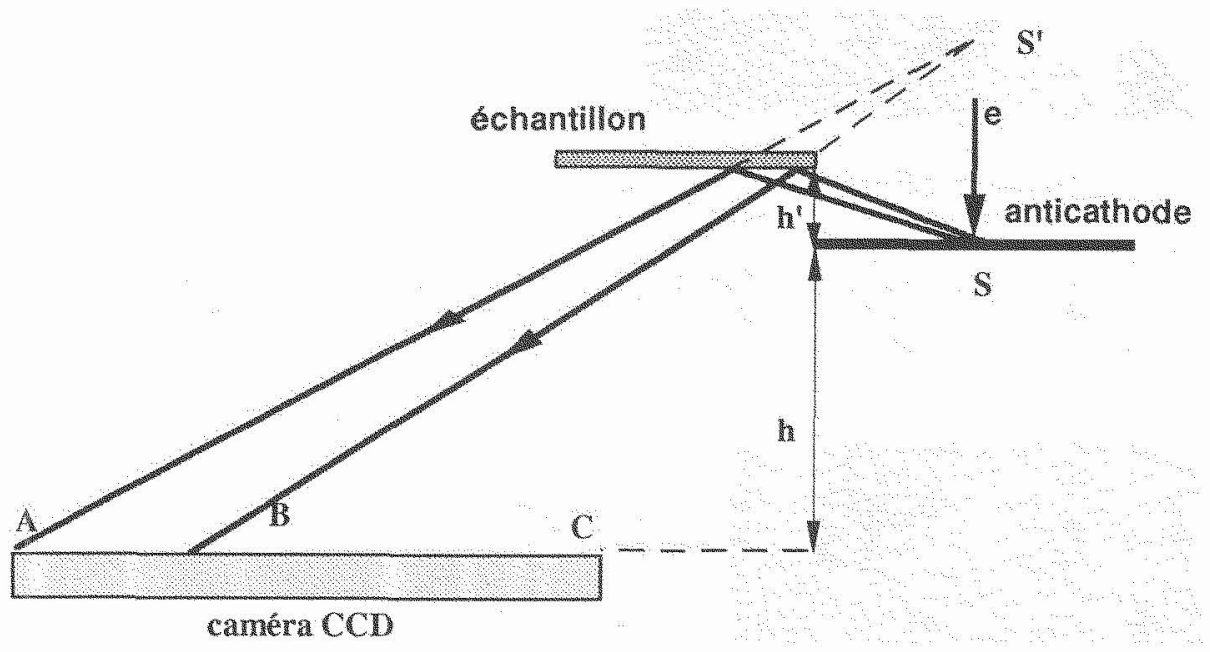

Figure 2 : Schéma de principe du dispositif retenu pour les expériences par réflexion (S : source $\mathrm{X} ; \mathrm{E}$ : échantillon; $C$ : caméra CCD); $h=550 \mu \mathrm{m}, \mathrm{h}^{\prime}=50 \mu \mathrm{m}$.

Schematic drawing of the experimetnal arrangement used in reflection mode experiments $(S: X$-ray source ; $\mathrm{E}$ : sample ; $:$ CCD camera); $\mathrm{h}=550 \mu \mathrm{m}, \mathrm{h}^{\prime}=50 \mu \mathrm{m}$.

\section{RESULTATS EXPERIMENTAUX PRELIMINAIRES}

Dans la dernière géométrie indiquée ci-dessus et en utilisant une anticathode de cuivre $\left(E_{0}=26 \mathrm{keV}\right)$ nous avons d'abord observé le diagramme de diffraction d'un cristal de silicium dont la surface est un plan (111).

Ce diagramme obtenu en 12 minutes est représenté sur la figure 3 . Il s'agit en fait d'un diagramme de Kossel obtenu par réflexion à l'aide probablement de la radiation $\mathrm{CuK} \alpha$ $(\lambda=1,54 \AA)$. Il faut observer que le faisceau utile sur l'échantillon n'est plus conique comme en transmission mais a plutôt une géométrie "en éventail" avec une divergence horizontale très grande puisque le demi-angle au sommet $\alpha / /$ des rayons extrêmes est tel que $\alpha / p 60^{\circ}$ mais avec une divergence verticale telle que $\alpha<1^{\circ}$. En fait le diagramme obtenu est beaucoup plus riche que ne le montre sa reproduction avec notamment l'existence de nombreuses tâches et lignes supplémentaires qui seraient en partie attribuables à la silice naturelle qui recouvre l'échantillon (voir schéma de droite Fig. 3).

Ayant obtenu très récemment ce résultat, l'indexation et l'analyse détaillées de ce diagramme est en cours. 

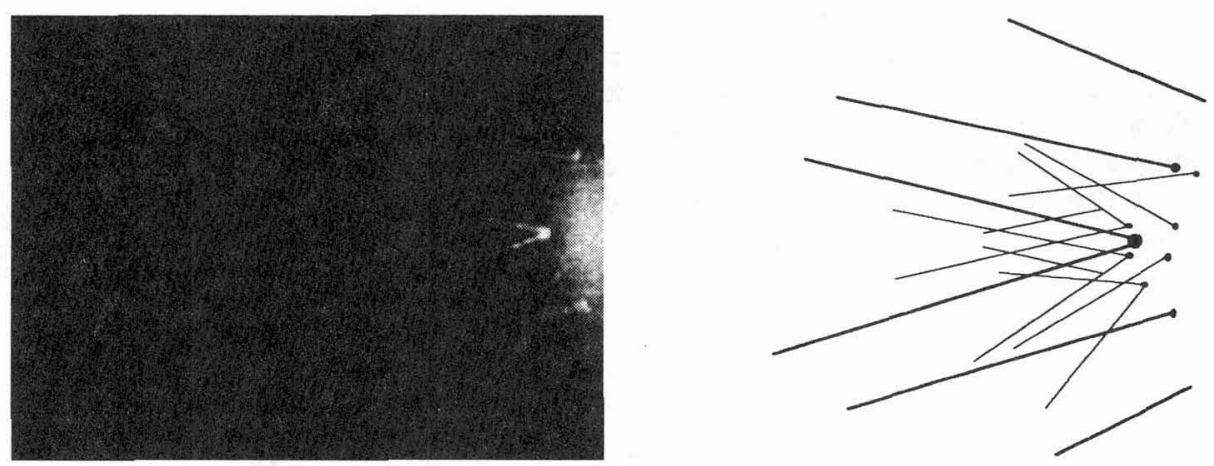

Figure 3 : Diagramme de diffraction obtenu par réflexion sur la surface Si (111) dans une géométrie en éventail. La source $\mathrm{X}$ et l'échantillon se situent à droite de la figure; pour plus de clarté, le dessin de gauche restitue la géométrie du diagramme.

Diffraction pattern obtained in the reflection mode on a $\mathrm{Si}(111)$ surface with a fan beam geometry. The specimen and the X-ray source are located in the right of the figure; for better view, the left drawing indicates the geometry of the diagram.
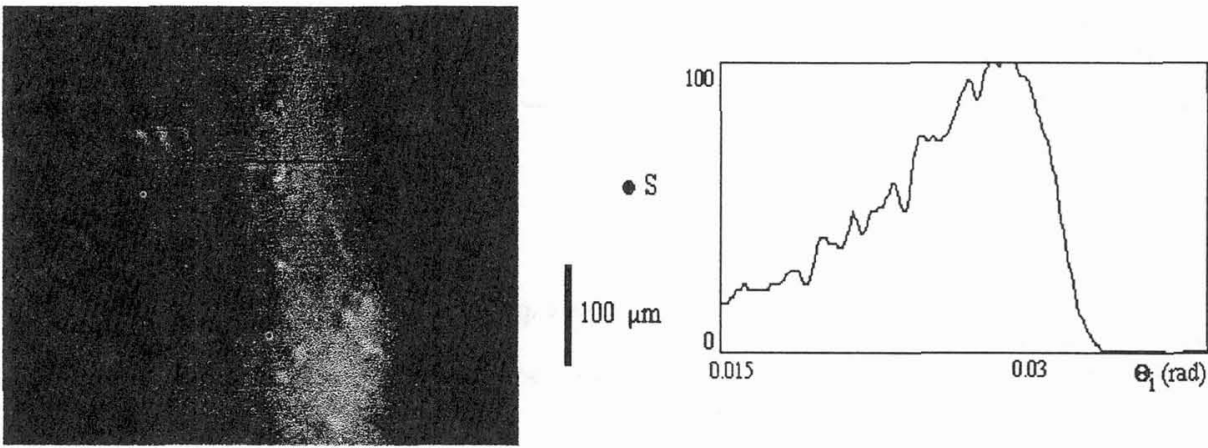

Figure 4: A gauche: Image $X$ de réflexion totale obtenue sur le cristal de silicium $\mathrm{Si}$ (111) à l'aide de la radiation CuL $\alpha$. A droite: profil d'intensité correspondant à la ligne noire du haut.

On the left: Total reflection X-ray image obtained on the silicon surface Si (111) with the help of the $\mathrm{CuL} \alpha$ radiation. On the right: Intensity profile corresponding to the black line shown on the top image.

Dans notre dispositif le faisceau d'électrons est balayable et la source $X$ est aisément déplaçable, il est donc très facile de changer l'angle moyen d'incidence des rayons $\mathrm{X}$ sur l'échantillon en décalant la position de l'impact des électrons incidents sur l'anticathode. En éloignant la source $X$ de l'objet on fait décroître l'angle d'incidence pour ainsi atteindre le domaine de réflexion totale des rayons $X$ mous générés par l'anticathode. La figure 4 représente une des images ainsi obtenues sur le cristal de silicium ayant conduit antérieurement au diagramme de diffraction de la Fig. 3. Les photons ayant contribué majoritairement à cette image sont probablement les photons $L \alpha$ du cuivre $(\lambda=13,3 \AA)$ avec un angle critique $\theta_{c}$ de l'ordre de $3 \cdot 10^{-2}$ radian. 
Les diagrammes et images obtenus étant numérisés par le système d'acquisition il est ensuite facile d'extraire de telles images ou diagrammes des profils d'intensité.

Un de ces profils est représenté au bas de la Fig. 3, où l'on peut identifier de droite à gauche d'abord l'absence de réflexion puis la zone de transition qui précède l'angle critique de réflexion totale. Au-delà du maximum au lieu du plateau attendu on observe une décroissance plus ou moins monotone de l'intensité ; nous attribuons cette décroissance à une décroissance de l'angle solide associée à l'éloignement de la source ou au fait que l'échantillon n'est pas strictement parallèle à la caméra CCD. Enfin les oscillations d'intensité correspondent aux granulosités de l'image qui est malheureusement affectée par la forme et la taille des grains du "phosphore" convertisseur. En effet si la géométrie présentée sur la Fig. 2 présente l'avantage de réduire l'anamorphose des images, elle conduit aussi à un éclairement du phosphore de la caméra à incidence quasi rasante ce qui a pour effet néfaste d'exalter les défauts topographiques du convertisseur.

Dans une dernière expérience, nous avons remplacé le cristal orienté (111) par un cristal orienté (100) qui avait l'avantage de présenter un défaut topographique sur sa surface. Nous avons pu mettre en évidence ce défaut sur les images de réflexion totale qui sont présentées sur les Fig. $5 a$ et $5 b$.

Ces deux images ont été obtenues successivement en déplaçant le faisceau d'électrons et donc la source $X$ vers le haut et parallèlement à l'arête de l'échantillon. Le déplacement en sens inverse du détail brillant visible sur les deux images nous permet de nous assurer que ce défaut est situé sur l'échantillon et non sur le convertisseur. On notera en particulier que ce défaut topographique de forme sensiblement rectangulaire est suivi d'une zone noire parallèle traduisant un déficit dans la réflexion des rayons X.

Comme pour le diagramme de diffraction de la Fig. 3, l'analyse détaillée de ces images récentes est en cours avec simultanément l'amélioration de notre montage (dont la géométrie précise est mal connue) et le remplacement du convertisseur par un cristal de YAG.

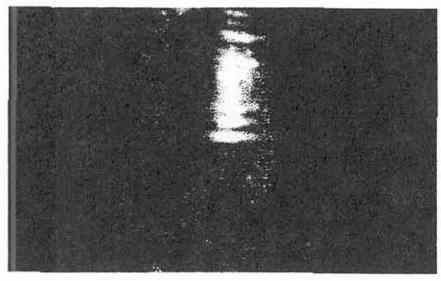

(a)
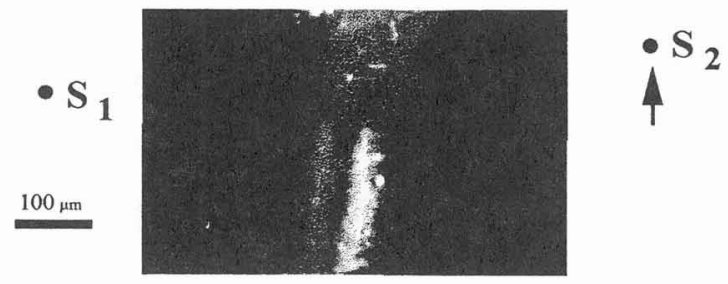

Figure 5 : Images $X$ de réflexion totale obtenue sur un cristal de silicium dont la surface est orientée (100). L'image de droite a été obtenue en déplaçant la source X, S, vers le haut (les autres conditions expérimentales sont inchangées par rapport à la Fig. 4).

$\mathrm{X}$-ray total reflexion images obtained on a $\mathrm{Si}(100)$ surface. The right image has been obtained by shifting the $X$-source upward. (The other experimental conditions are the same as for Fig. 4). 


\section{CONCLUSION}

Nous avons transformé un microscope électronique à balayage en microscope microtomographe $X$ par l'adjonction d'une caméra $\mathrm{CCD}$. En modifiant ensuite la position relative de la source $\mathrm{X}$ et de l'échantillon par rapport à cette caméra nous avons pu compléter les possibilités de cet instrument de laboratoire par l'acquisition de diagrammes de diffraction $\mathbf{X}$ (à incidence rasante et faisceau divergent) et par l'obtention des premières images de microscopie $\mathrm{X}$ à réflexion totale. L'analyse détaillée de ces résultats est en cours et nous explorons les possibilités offertes par une géométrie originale mise en oeuvre dans un équipement de laboratoire très simple. On aura en effet noté que les dispositifs utilisant des faisceaux $\mathrm{X}$ incidents parallèles (comme la radiation synchrotron) ou collimatés ne permettent pas d'obtenir des diagrammes de Kossel, et surtout, que les images éventuelles de rélexion totale n'auraient alors qu'un grandissement unitaire.

\section{Références}

[1] Nevot L. et Croce P., Rev. Phys. Appl. 15 (1980) 761-779

[2] Parratt L.G., Phys. Rev. 95 (1954) 359-369

[3] de Boer D.K.G., Phys. Rev. B, 49 (1994) 5817-5820

[4] Holy V., Kubena J., Ohlidal I, Lischha K, Plotz W, Phys. Rev. B, 47 (1993) 15896-15903

[5] Belorgey O et Benattar J.J., Phys. Rev. Lett. 66 (1991) 313

[6] Marra W.C., Eisenberger P., Cho AV. J. Appl. Phys. 50 (1979) 6927

[7] de Boer D.K.G, Leenaers A.J.G, van der Hoogenhof V.W., X ray Spectr.24(1995) 91-102.

[8] Erre D. et al, Inst. Phys. Conf. Series 130 (1993) 567-570

[9] Cazaux J. et al, Inst. Phys. Conf. Series, Proceeding EMAG 95, à paraître.

[10] Cazaux J. et al, J. Phys. IV France, C7, supplément au J. Phys. III, 3 (1993) 2099-2104

[11] Elhila $\mathrm{H}$. et al, ce congrès

[12] Rondot $S$. et al, ce congrès

[13] Yakowitz H, Adv. Electron Electron. Phys. (1968) 361-431 